\title{
Beyond a single patch: local and regional processes explain diversity patterns in a seagrass epifaunal metacommunity
}

Keila A Stark ${ }^{1 *}$, Patrick L Thompson ${ }^{1}$, Jennifer Yakimishyn ${ }^{2}$, Lynn Lee ${ }^{3}$, Emily M Adamczyk ${ }^{1}$, Margot Hessing-Lewis ${ }^{4}$, Mary I O'Connor ${ }^{1}$

1. Biodiversity Research Centre and Department of Zoology

University of British Columbia

10 Vancouver, BC, Canada

2. Pacific Rim National Park Reserve

13 P.O. Box 280

14 Ucluelet, BC, Canada

3. Gwaii Haanas National Park Reserve, National Marine Conservation Area Reserve, and Haida Heritage Site 60 Second Beach Road

Skidegate, BC, Canada

4. Hakai Institute

PO Box 309

Heriot Bay, BC, Canada

*Corresponding author: keilastark@,zoology.ubc.ca

Abstract: 251

Main text: 5000

Number of references: 68

Number of figures: 3

Data accessibility: Data will be made permanently available on Dryad should the manuscript be accepted. All data and code for the analysis can be currently found at

37 https://github.com/keilast/HMS-Seagrass.

Competing interests: We have no competing interests.

\section{Acknowledgements}

42 KAS and MIO conceived of the study. JY provided samples from IN, DK, and EB. LL provided 43 samples from RA and HL. EMA and MHL provided samples from TN, TB, CI, and CS. KAS 
44 collected and processed samples, analyzed data, and wrote the first manuscript draft. PLT

45 provided recommendations for the analysis. KAS, PLT, and MIO contributed to writing the

46 manuscript. This research was financially supported by a UBC Zoology SURE Grant, and

47 NSERC Discovery Grants, Canadian Foundation for Innovation (CFI), and is sponsored by the

48 NSERC Canadian Healthy Oceans Network and its Partners: Department of Fisheries and

49 Oceans Canada and INREST (representing the Port of Sept-Îles and City of Sept-Îles). PLT is

50 supported by NSERC and Killam postdoctoral fellowships. We are grateful to the Tula

51 Foundation for providing logistical support and facilities during Calvert Island field collections.

52 Special thanks go to Michelle Paleczny and Gulf Islands National Park Reserve for boat and

53 laboratory space, and staff, students, and volunteers from Pacific Rim National Park Reserve for

54 their assistance collecting eelgrass. We thank Minako Ito, Ariane Comeau, Rob Underhill, Abbie

55 Sherwood, Giordano Bua for assistance with samples and Coreen Forbes, Matt Whalen, and

56 anonymous reviewers for feedback on the manuscript.

\section{Abstract}

59 Ecological communities are jointly structured by dispersal, density-independent responses to

60 environmental conditions and density-dependent biotic interactions. Metacommunity ecology

61 provides a framework for understanding how these processes combine to determine community

62 composition among local sites that are regionally connected through dispersal. In 17 temperate

63 seagrass meadows along the British Columbia coast, we tested the hypothesis that eelgrass

64 (Zostera marina L.) epifaunal invertebrate assemblages are influenced by local environmental

65 conditions, but that high dispersal rates at larger spatial scales dampen effects of environmental

66 differences. We used hierarchical joint species distribution modelling to understand the 
67 contribution of environmental conditions, spatial distance between meadows, and species co-

68 occurrences to epifaunal invertebrate abundance and distribution across the region. We found

69 that patterns of taxonomic compositional similarity among meadows were inconsistent with

70 dispersal limitation and meadows in the same region were often no more similar to each other

71 than meadows over $1000 \mathrm{~km}$ away. Abiotic environmental conditions (temperature, dissolved

72 oxygen) explained a small fraction of variation in taxonomic abundances patterns across the

73 region. We found novel co-occurrence patterns among taxa that could not be explained by shared

74 responses to environmental gradients, suggesting the possibility that interspecific interactions

75 influence seagrass invertebrate abundance and distribution. Our results add to mounting evidence

76 that suggests that the biodiversity and ecosystem functions provided by seagrass meadows reflect

77 ecological processes occurring both within meadows and across seascapes, and suggest that

78 management of eelgrass habitat for biodiversity may be most effective when both local and

79 regional processes are considered.

81 Key words: Hierarchical Modelling of Species Communities, priority effects, dispersal, niche

82 filtering, British Columbia, Zostera marina

\section{Introduction}

85 Understanding how local environmental conditions, regional connectivity by dispersal and

86 biotic interactions jointly structure the composition of communities is a central challenge in

87 ecology (Ricklefs and Schluter 2003, Vellend 2010, Leibold and Chase 2017). Metacommunity

88 ecology (Leibold et al. 2004, Leibold and Chase 2017, Thompson et al. 2020) offers a

89 framework for understanding community assembly processes across spatial scales. In recent 
90 years, applications of the metacommunity framework have emphasized the underlying processes

91 that give rise to abundance and diversity patterns (Brown et al. 2017, Leibold and Chase 2018,

92 Thompson et al 2020). Thompson et al. (2020) framed the metacommunity concept based on

93 three fundamental processes that together govern the dynamics of populations and communities:

94 1) Density-independent responses to environmental conditions, 2) Density-dependent biotic

95 interactions (ie. inter and intra-specific competition, predation or facilitation influencing

96 population growth and co-existence), and 3) Dispersal influencing connectivity across a

97 landscape or seascape. We apply this framework to understand the contribution of these

98 processes to a seagrass-associated invertebrate metacommunity.

Many coastal environments that host high biodiversity occupy spatially structured habitats

100 (e.g. coral reefs, kelp forests, seagrass meadows), and many marine species have dispersing life

101 histories that link populations in distinct habitat patches. The importance of interspecific

102 interactions (Berlow 1999, Sala and Graham 2002), and dispersal-driven population dynamics

103 (Levin and Paine 1974, Gaines and Roughgarden 1985) in coastal and marine communities has

104 long been recognized. These combined roles of dispersal, the local environment, and biotic

105 interactions suggest patterns of marine biodiversity reflect metacommunity processes (Boström

106 et al. 2006).

107 Seagrasses are foundation species that support high productivity and faunal diversity (Orth et

108 al. 1984, Duffy et al. 2015). They form meadows separated by deeper water, un-vegetated

109 seafloor, or other vegetated habitats, and associated fauna disperse among meadows (Boström et

110 al. 2006, 2011). Diverse invertebrate assemblages including snails, amphipods, isopods, and

111 polychaete worms live among seagrass leaves, providing food sources for larger invertebrates,

112 fish, and birds (Best and Stachowicz 2014, Huang et al. 2015). The grazers within this group 
113 consume detritus, macroalgae, or the seagrass itself (Valentine and Heck 1999), and many taxa

114 exert top-down control on epiphytic microalgae that compete with the seagrass for light and

115 nutrients (Sand-Jensen 1977, Duffy and Stachowicz 2006). Seagrass-associated epifauna exhibit

116 a range of dispersal modes (fast swimming by isopods; slow crawling by snails; permanent

117 attachment in bivalves) and reproductive strategies (brooding versus broadcast spawning) which

118 influence dispersal rates and the distances over which meadows are demographically connected.

119 Seagrass meadows occur in a wide range of temperature, salinity and hydrodynamic

120 environments, such that meadows may differ in their suitability for various invertebrate taxa

121 (Yamada et al. 2007, 2014). Evidence for the importance of environmental filtering -- or the

122 tendency for taxa to exist where local environmental conditions are ideal -- has been reported in

123 several seagrass-associated fish and invertebrate systems (Baden et al. 2010, Robinson et al.

124 2011, Iacarella et al. 2018).

125 Though interspecific interactions among seagrass-associated invertebrates are not extensively

126 documented, there is some evidence of competition and predation influencing community

127 structure (Nelson 1979, Best and Stachowicz 2014). Predation can reduce abundances of

128 vulnerable invertebrate taxa, allowing others to increase in abundance (Nelson 1979, Baden et al.

129 2010, Best and Stachowicz 2014, Amundrud et al. 2015, Huang et al. 2015). Despite some

130 evidence for competition among epiphytic grazers for shared food sources (Edgar 1990, Bruno

131 and O'Connor 2005) there is no evidence of competitive dominance to the point of exclusion in

132 the field (Nelson 1979, Best and Stachowicz 2014).

133 While research in past decades has found evidence for direct and indirect influences of local

134 characteristics such as habitat complexity (Orth et al. 1984), primary biomass (Cébrian and

135 Duarte 1998, Gullström et al. 2012), and nutrient availability (Virnstein and Howard 1987) on 
136 faunal diversity and abundance, recent studies have focused more on regional scale or multi-

137 meadow processes such as dispersal (Whippo et al. 2018, Lefcheck et al. 2016, Stier et al. 2019,

138 Yeager et al. 2019), and have found that the influence of local-scale factors are often overriden

139 by regional patterns of inter-meadow connectivity (Lefcheck et al. 2016, Stier et al. 2019, Yeager

140 et al. 2019). This pattern has been attributed to relatively rapid life histories and high inter-

141 meadow dispersal in seagrass-associated organisms (Lefcheck et al. 2016).

142 We designed this study to better understand the contributions of dispersal, local

143 environmental conditions, and interspecific interactions with the largest spatially explicit (in

144 geographic extent) dataset of seagrass metacommunity diversity. Drawing upon metacommunity

145 theory, we tested the hypotheses that: (H1) Spatial distance per se within a region does not

146 confer community dissimilarity because meadows are well-connected by dispersal, even at larger

147 spatial scales (dispersal limitation hypothesis); (H2) Differences in local-scale environmental

148 factors such habitat structure, temperature, and salinity drive some differences in invertebrate

149 presence and abundance owing to differences in environmental niches among taxa, however the

150 contribution of environmental factors is smaller than the contribution of spatial distance/

151 dispersal (environmental filtering hypothesis); (H3) No clear patterns of spatial co-occurrence

152 among epifaunal species emerge because high dispersal and environmental responses override

153 signatures of biotic interactions (predation, competition) that would produce such patterns (biotic

154 interactions hypothesis).

156 Methods

157 Study sites

158 We sampled epifaunal invertebrate diversity in 17 Zostera marina (L.) meadows 
159 spanning the entire coast of British Columbia (approximately $1000 \mathrm{~km}$ distance) from late June

160 through August of 2017. Within five regions that each contain numerous eelgrass meadows, we

161 sampled a few meadows for our study (Fig. 1a): Haida Gwaii (two meadows); the central coast

162 of BC near Calvert Island (four meadows); southern Clayoquot Sound (three meadows); Barkley

163 Sound on the West coast of Vancouver Island (three meadows); the Southern Gulf Islands (five

164 meadows). Meadows varied in environmental conditions, such as seagrass shoot size and

165 density, hydrological regimes, and freshwater outflow influencing salinity.

166

167 Field sampling

168 We sampled three subtidal meadows with SCUBA in the Barkley Sound (SA, DC, RB)

169 and Central Coast (TB, TB, CI, CS) regions. All others were sampled by wading or snorkeling

170 at low tide. We conducted collections within a six-week period of peak seagrass growth. We

171 used $0.25 \mathrm{~m} \times 0.25 \mathrm{~m}$ quadrats to collect all above-ground eelgrass, epifaunal invertebrates,

172 eelgrass detritus, and macroalgae in each sample, following Whalen et al. (2013). Six quadrats

173 were arranged in a $15 \mathrm{~m} \mathrm{x} 30 \mathrm{~m}$ array (Appendix 1: Fig. S1) in the middle of the meadow to

174 avoid edge effects. We uprooted eelgrass shoots at the first node, leaving rhizomes to avoid

175 sampling infauna that live in the sediments, removed all other above-ground biomass (detritus,

176 macroalgae, associated epifauna) within each quadrat by hand, and immediately placed all the

177 contents into a $300 \mu \mathrm{m}$ mesh or plastic Ziploc bag for transport to the lab.

\section{Environmental data}

We acquired water quality data from regional data sources. The abiotic water quality data

181 were annual means pulled from Bio-ORACLE (Assis et al. 2018), including oxygen, nitrates, 
182 phosphates, silicates, salinity, maximum current velocity and sea surface temperature. Bio-

183 ORACLE is a database of marine environmental layers gathered from several satellite and in-situ

184 sources at a spatial resolution of $0.08^{\circ}$, and has been shown to accurately model distributions of

185 shallow-water invertebrate species (Tyberghein et al. 2012, Assis et al. 2018). Sea surface

186 temperature layers were taken from the Aqua-MODIS satellite, and values for nitrates, dissolved

187 oxygen, and salinity were interpolated from in-situ measurements reported in the World Ocean

188 Database (Tyberghein et al. 2012). All meadows were situated in distinct spatial cells, and thus

189 had distinct values for the aforementioned variables. We excluded phosphate and silicate

190 concentrations from our final analysis, as they strongly covaried with nitrates.

191 In each quadrat sample, we measured four biotic attributes summarizing habitat structure

192 and food availability: eelgrass leaf area index (LAI), and eelgrass, eelgrass detritus, and algal dry

193 mass. To quantify LAI, we measured leaf length, width, and blade number in five haphazardly-

194 chosen shoots from each quadrat, and multiplied the average blade area per shoot by the quadrat-

195 level shoot density. We dried eelgrass, detritus, and macroalgae in a desiccator oven $\left(60^{\circ} \mathrm{C}\right.$ for

19648 hours) to measure ash-free dry mass.

Invertebrate identification

Immediately after collection, we rinsed eelgrass shoots with fresh water, passing the

200 water through a $500 \mu \mathrm{m}$ sieve to remove epifaunal invertebrates. Invertebrates were preserved in

$20195 \%$ EtOH for identification with light microscopy (10x magnification). We identified

202 invertebrates to the lowest taxonomic level possible using keys in the Light and Smith manual

203 (Carlton 2007) and Kozloff (1996). In many cases, the lowest taxonomic resolution possible was

204 family or genus, therefore our biodiversity survey likely underestimates full species diversity. 
206 Analysis

All statistical analyses were conducted in R (version 3.6.0; R Development Core Team

208 2019. We followed the Hierarchical Modelling of Species Communities (HMSC) framework

209 (Ovaskainen et al. 2017) using the 'Hmsc-R' package (Tikhonov et al. 2019) to fit a hierarchical

210 joint species distribution model (JDSM) with Bayesian inference. The framework uses traditional

211 single-species distribution modelling by estimating species responses (presence or abundance) to

212 environmental covariates across samples, but does so for all species simultaneously. It can use

213 residual variation in occurrences to infer species co-occurrence patterns that do not result from

214 shared responses to environmental covariates in the model (Ovaskainen et al. 2017). HMSC can

215 also account for spatially hierarchical sampling structures. In these ways, the HMSC framework

216 overcomes statistical limitations of previous methods used in metacommunity studies (Gilbert and

217 Bennett 2010, Tuomisto et al. 2012, Brown et al. 2017).

219 that describes the abundance of species $j$ (where $j=1 \ldots n$ ), where $y_{i j}$ is the abundance of species $j$

220 in sample $i, D$ is the statistical distribution of the abundance data (Poisson distribution in this study),

$221 L_{\mathrm{ij}}$ is the linear predictor to link species' presence with environmental covariates, and $\sigma_{j}^{2}$ is a

222 variance term for the abundance of species $j$ :

$$
y_{i j}{ }^{D} \sim D\left(L_{i j}, \sigma_{j}^{2}\right)
$$

224

225 The linear predictor $L_{\mathrm{ij}}$ is described by fixed $(F)$ and random $(R)$ effect terms:

$$
L_{i j}=L^{F_{i j}}+L^{R}{ }_{i j},
$$


The fixed effect term $L^{F}{ }_{i j}$ is modelled as a regression:

$$
L^{F}{ }_{i j}=c_{j k} x_{i k}
$$

where $x_{i k}$ represents the measured value for environmental covariate $k$ for a given sample $i$ (e.g.,

233 biomass in quadrat $i$ ), and parameter $\beta$ represents the relationship between environmental 234 covariate $k$, and the abundance of species $j$.

235 The random effect term $L^{R}{ }_{i j}$, captures the variation in species abundances that cannot be explained 236 by the measured covariates. It is further denoted as:

$$
L^{R}{ }_{i j}=\varepsilon_{i j} Q_{+} \varepsilon_{i j}{ }^{S}+\varepsilon_{i j}^{R}+\varepsilon_{i j}^{D}
$$

238 where the terms describe three random effects associated with our spatially nested sampling 239 scheme $\left(\varepsilon_{i j} Q, \varepsilon_{i j}^{S}, \varepsilon_{i j}^{R}\right.$ for Quadrat (sample), Site (meadow), and Region), as well as a fourth spatially 240 explicit random effect (spatial autocorrelation in species abundances). The spatially explicit 241 random effect $\varepsilon_{i j}{ }^{D}$ was calculated with latent factor analysis that takes into account spatial distance 242 between all pairwise combinations of samples (see Ovaskainen et al. 2016a for details).

244 distributions $\varepsilon_{i j} Q \sim N\left(0, \Omega^{Q}\right), \varepsilon_{i j}^{S} \sim N\left(0, \Omega^{S}\right), \varepsilon_{i j}^{R} \sim N\left(0, \Omega^{R}\right)$. Variance terms $\Omega^{Q}, \Omega^{S}, \Omega^{R}$, are 245 variance-covariance matrices (square matrices containing all taxa in the model), where the 246 diagonal elements give species-specific residual variance in occurrences among samples, and the 247 off-diagonal elements give residual co-variances between species pairs. The term "residual" refers 248 to the fact that it is the variance unexplained by environmental covariates in the fixed effects 249 predictor described above. These variance-covariance matrices $\left(\Omega^{Q}, \Omega^{S}, \Omega^{R}\right.$, and $\left.\Omega^{D}\right)$ are 250 parameters estimated using a latent variable approach described in Ovaskainen et al. (2016b). They 
251 were used to represent co-occurrence matrix R, where $j_{1}$ and $j_{2}$ refer to two species within the

252 model, and $\mathrm{R}_{j 1 j 2}=\Omega_{j 1 j 1} / \sqrt{\Omega_{j 1 j 1} \Omega_{j 2 j 2}}$. R describes the extent to which a given species pair co-

253 occurs more positively or negatively than by chance, after controlling for possible shared responses

254 to the same environmental covariates.

We estimated parameters with Markov chain Monte Carlo. Markov chains were run to

256200000 iterations, a burn-in length of 1000 iterations, and was thinned to retain every $10^{\text {th }}$

257 sample of the posterior distributions. We confirmed that Markov chains were well-mixed by

258 visually inspecting trace plots. Estimates of parameter $\beta$ (environmental responses across taxa)

259 and $\Omega$ (variance-covariance matrices used to visualize species co-occurrence patterns

260 independent of the environment) were extracted as $95 \%$ credible intervals. We evaluated the

261 model fit ( $\mathrm{R}^{2}$ and Root-Mean-Square Error or RMSE) using 4-fold cross-validation.

To test our three hypotheses, we visualized different aspects of the parametrized JDSM.

263 To test for dispersal limitation (H1), we looked for increased pairwise community dissimilarity

264 among samples with increased pairwise spatial distance. We predicted species abundances in

265 every sample using our trained model, then calculated pairwise Bray-Curtis dissimilarity index

266 (Bray and Curtis 1957) on these predicted abundances. We then conducted log-linear regression

267 of predicted pairwise dissimilarity against pairwise spatial distance $(\mathrm{km})$ on a logarithmic scale.

268 Increased community dissimilarity could reflect dispersal limitation due to prohibitively far

269 dispersal distances, or due to increasing differences in environmental conditions with increasing

270 spatial scale. To distinguish between these possibilities, we compared a regression of predicted

271 dissimilarity based on the full JDSM (including environmental covariates) with the predicted

272 dissimilarity calculated from the same trained model, but with no effect of the environment (all

273 environmental covariates were set to their mean values). 
To test the environmental filtering hypothesis $(\mathrm{H} 2)$, we analyzed $\beta$ for each combination

275 of species and environmental covariate to determine whether they had a positive or negative

276 relationship. We also partitioned the variance in abundance explained by environmental

277 covariates, spatial autocorrelation, and sampling design random effects.

278 To test the biotic interactions hypothesis (H3), we represented co-occurrence matrix R as

279 a correlation plot for each random effect level, demonstrating the extent to which species pairs

280 co-occurred more negatively or positively than predicted by their modelled correlation with

281 environmental covariates at each spatial scale (Ovaskainen et al. 2017, Aivelo and Norberg

282 2018).

283

284 Results

285 Taxonomic abundance and distribution patterns

286 We identified 52282 individuals representing at least 50 taxa across the region

287 (Appendix 2: Table 2). Of these, 3\% of individuals were bivalves, 12\% were gastropods (snails

288 and limpets), 14\% were copepods, $41 \%$ were polychaetes (most of which were calcifying

289 polychaetes Spirorbis sp.), 13\% were gammarid amphipods, 6\% were caprellid amphipods, and

290 the remaining $11 \%$ included other crustaceans (isopods, tanaids, cumaceans, crabs, shrimp).

291 These taxa span several phyla, diet types (herbivores, detritivores, suspension feeders), and

292 dispersal strategies (brooding, broadcast spawning). Mean meadow-level taxonomic richness

293 was 20 taxa per $0.0625 \mathrm{~m}^{2}$ of seagrass area.

294 Several taxa were present at all 17 meadows: harpacticoid copepods Porcellidium sp.

295 (4\% of all individuals across the entire region), snails Lacuna spp. (7\%), and tanaids Leptochelia

296 sp. (10\%). We found genera Mytilus sp. (mussel) and Nereis sp. (polychaete worm) in all 
meadows, however we were not confident the same species were present at each meadow. Ten taxa were present in every region, but not necessarily every meadow: isopod Pentidotea resecata, gammarid amphipods Monocorophium insidiosum, Ampithoe valida, Pontogeneia

300 rostrata, Ampithoe dalli, Aoroides spp., snails Amphissa columbiana and Alia carinata, and

301 limpet Lottia pelta.

We excluded rare species (those found in fewer than $5 \%$ of samples, or fewer than 5 out S2). This was necessary to avoid statistically over-inflating the importance of model covariates.

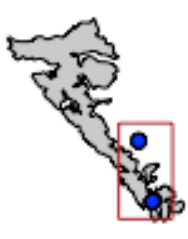

Haida Gwaii
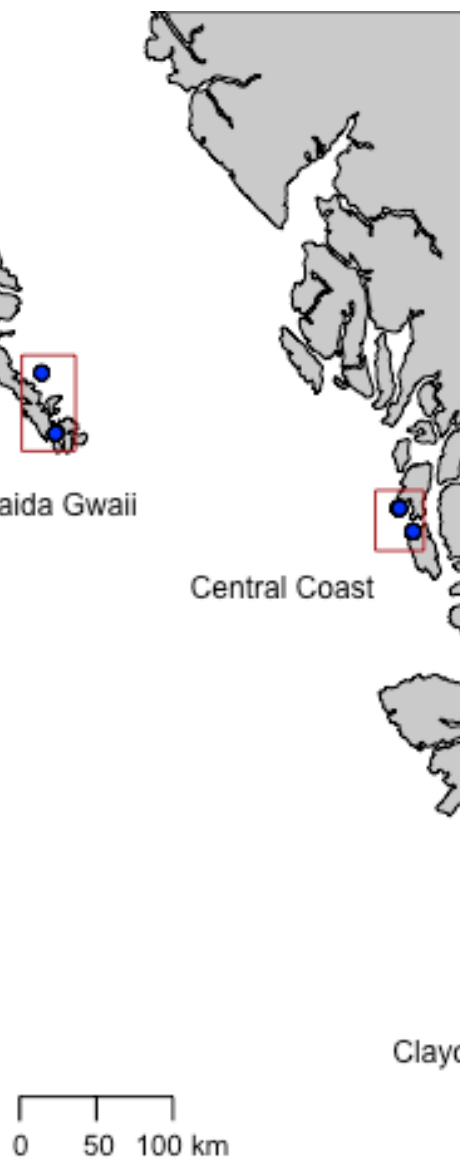

Central Coast
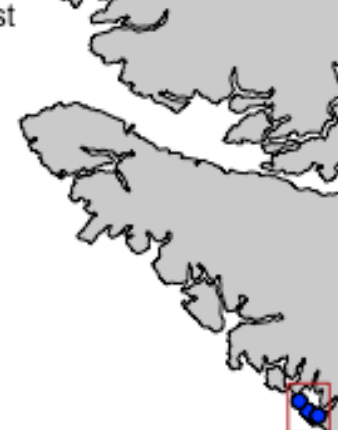

Clayoquot Sound

Barkley Sound

Gulf Islands 
309 Spatial connectivity via dispersal driving community similarity

310 The highest Bray-Curtis dissimilarity value was 0.99 for a pair of sites spaced $787 \mathrm{~km}$

311 apart (HL in Haida Gwaii and SA in Clayoquot Sound). The two communities at the highest

312 pairwise distance (997 km, RA in Haida Gwaii and SS in the Gulf Islands) had a dissimilarity

313 index of 0.8 . The lowest dissimilarity index value was 0.59 at a pairwise spatial distance of 0.6

$314 \mathrm{~km}$ (LH and SS in the Gulf Islands). A log-linear regression of Bray-Curtis dissimilarity

315 calculated from predicted abundances using the JDSM showed that community dissimilarity

316 increased with increased spatial distance (Fig. 1b, $y=0.75+0.023 \log (x), \mathrm{n}=272, \mathrm{R}^{2}=0.16$ ).

317 The maximum and minimim pairwise dissimilarity values were reduced when the effect of the

318 environment was removed $\left(y=0.74+0.021 \log (x), \mathrm{n}=272, \mathrm{R}^{2}=0.07\right)$, suggesting

319 environmental variables drive some dissimilarity in species composition with increased spatial 320 distance. 


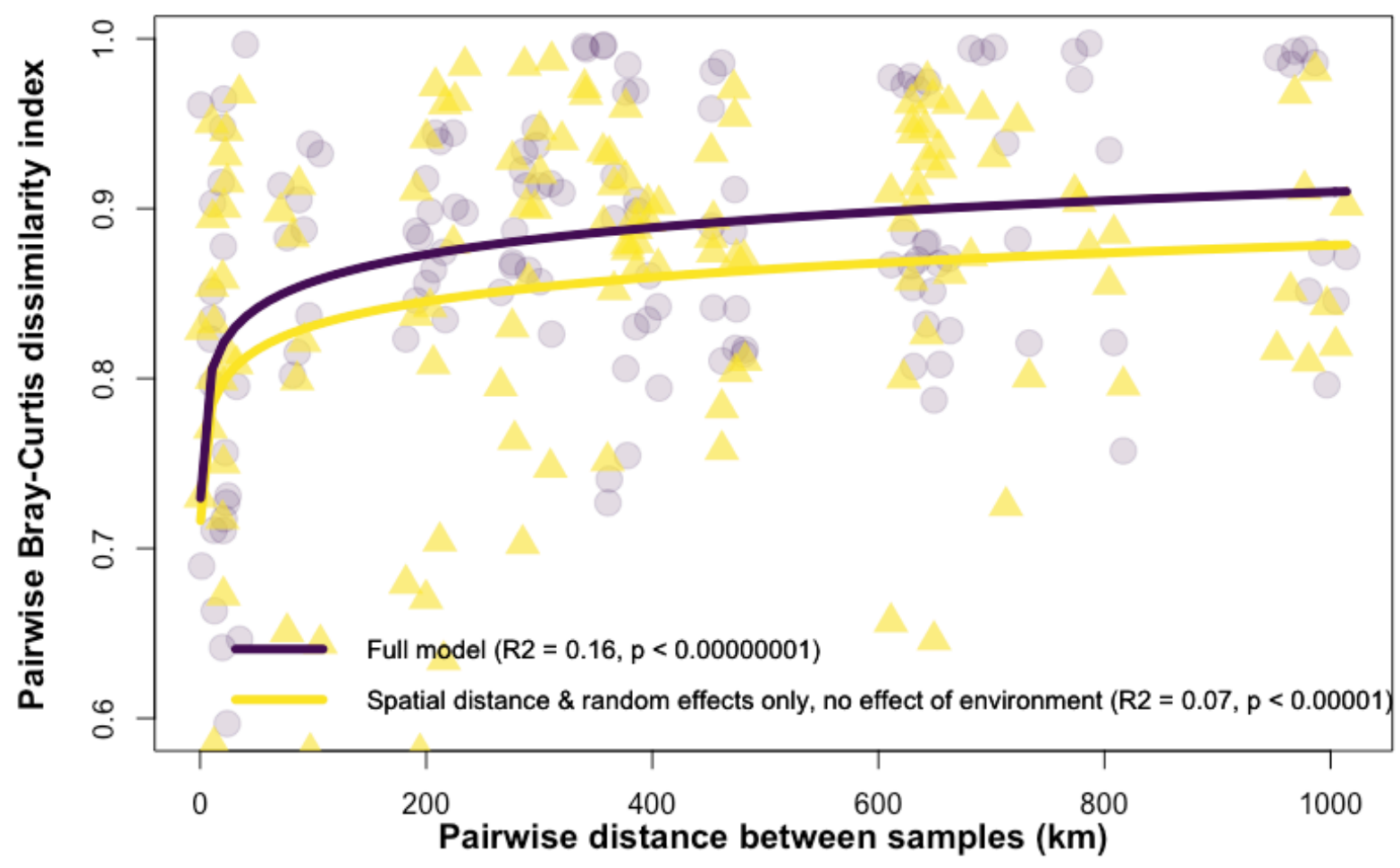

Figure 1b. Bray-Curtis pairwise community dissimilarity index as a function of spatial distance (Euclidean) between every pairwise combination of meadows in our study. The purple points and line show pairwise dissimilarity predicted by the full joint species distribution model using the original environmental and spatial data. The yellow points and line show pairwise dissimilarity predicted by the same trained model, but the effect of all environmental variables has been removed (set to their mean values). The yellow points and line show calculated pairwise dissimilarity based on the raw abundance data.

331 The assessment of model fit and variance partitioning analysis both showed that the importance

332 of environmental covariates differed among taxa, and that overall the environment explained

333 relatively a low proportion of variation in species abundances across the region (Fig 2a). While

334 the mean $\mathrm{R}^{2}$ across individual species distribution models was 0.27 , individual $\mathrm{R}^{2}$ values varied

335 (0 to 0.68, Appendix 1: Table A2) indicating variability in model fit. 


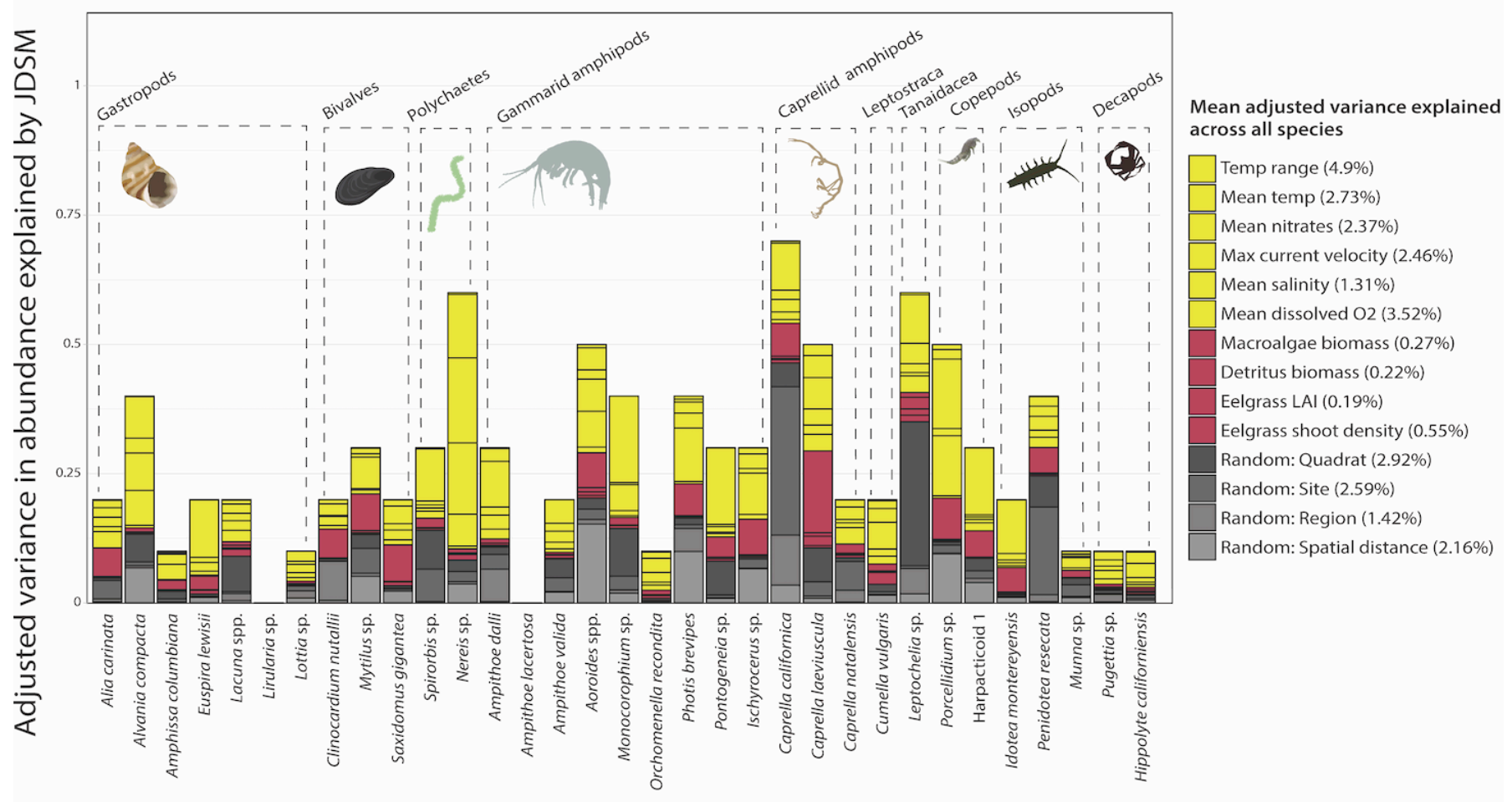

Species

337 Figure 2a. Variation partitioning of fixed and random effects within the joint species distribution model. Variances explained have been adjusted to reflect the model fit (Multiplied by Pseudo $\mathrm{R}^{2}$ ). An unadjusted version of the variance partitioning analysis can be found in Appendix 1, Fig. S3 of the Supplementary Information. Dashed lines indicate broad invertebrate taxonomic groupings. Grey cells represent the contribution of random effects associated with the sampling design. Yellow cells represent abiotic water quality covariates. Pink cells represent biotic covariates (food availability, habitat structure). The percentages next to the legend labels indicate mean variation explained by that covariate/ random effect across all species distribution models.

344 (Fig. 2a). Mean and range of sea surface temperatures and dissolved oxygen explaining the

bars, Fig. 2a). The importance of some environmental covariates differed markedly among taxa;

348 for example, nitrate levels explained approximately $27 \%$ of variance in the abundance of

349 gastropod Alvania compacta across meadows, but only 5\% of variance in gastropod Alia 
351 was $2.2 \%$ (pale gray bars, Fig. 2a), and other hierarchical random effects associated with the

352 sampling design explained 2.92\% (quadrat-level), 2.59 (meadow or site-level), and 1.42\%

353 (region-level) of variation.

354 The strength and directionality of responses to environmental variables also differed

355 among taxa, based on inspection of the $\beta$ parameters that estimate the relationships between

356 environmental covariates and species abundances (Fig. 2b). None of the taxa in our study

357 showed statistically supported responses ( $\beta$ within $95 \%$ credible interval) to all environmental

358 variables. The few that showed a significant response to eelgrass LAI responded positively

359 (higher LAI meant higher abundances). Thirteen taxa responded negatively to higher temperature

360 ranges, and two responded positively (Fig. 2b). The majority of taxa (22 out of 33) had

361 significant responses to dissolved oxygen levels. Of these, 8 responded negatively (high

362 dissolved $\mathrm{O}_{2}$ yields lower abundances), and 14 responded positively (high dissolved $\mathrm{O}_{2}$ yields

363 lower abundances). 


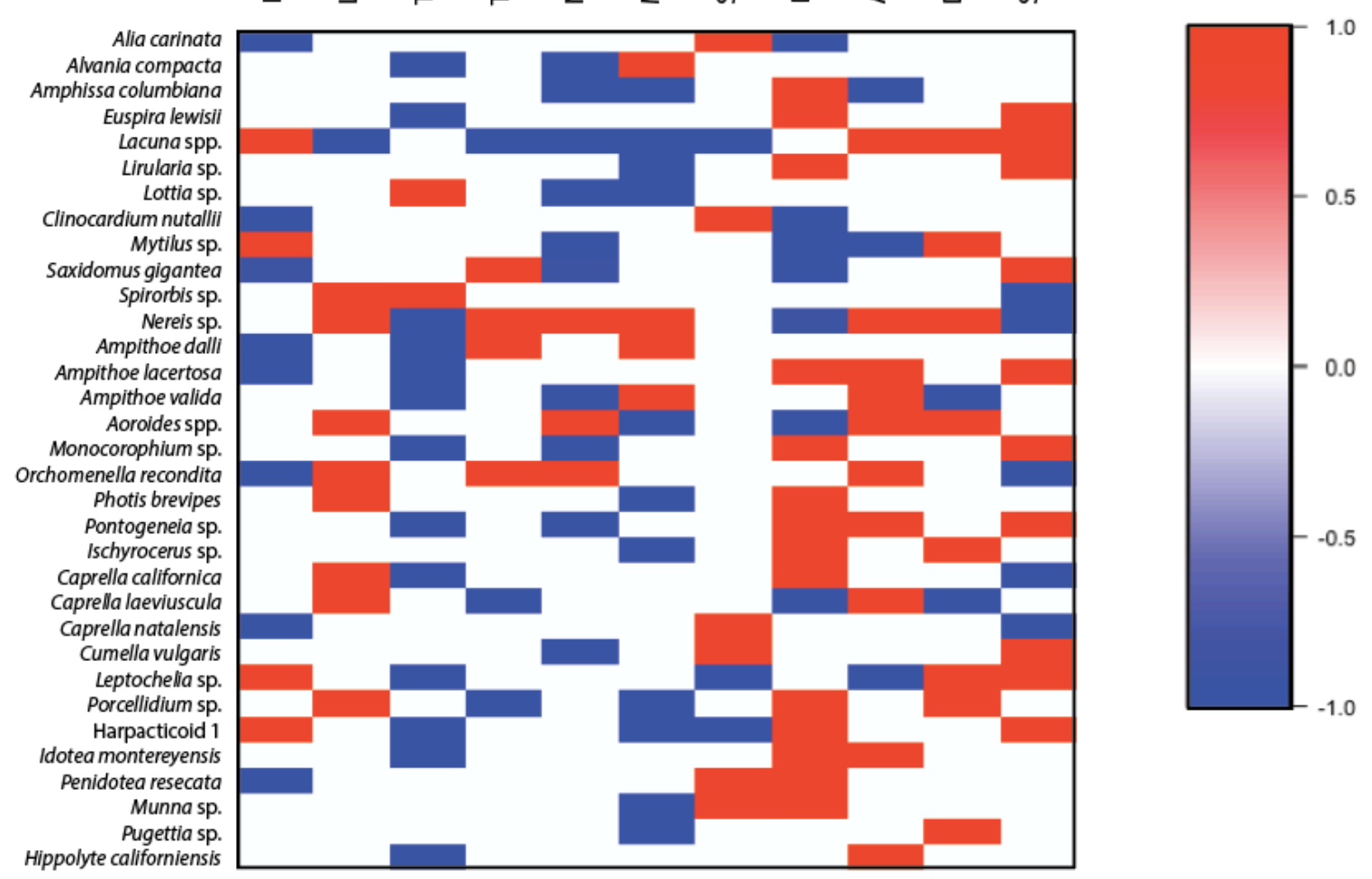

365 Figure 2b. Heat plot summarizing beta $(\beta)$ parameters, or responses to all environmental covariates in the joint species distribution model. Coloured cells indicate that the response is statistically supported ( $\beta$ falls within $95 \%$ credible interval). Red cells indicate positive responses to a given environmental covariate (higher abundance correspond with higher values of the environmental covariate), whereas blue cells indicate negative responses (lower abundance with higher values of the environmental covariate).

Co-occurrence patterns were less strong at the between-quadrat and between-region

373 spatial scales (Fig 3a, 3c). In comparisons among quadrats, we observed more positive species

374 co-occurrences than negative co-occurences (Fig. 3a). In comparisons among meadows, we

375 identified, post hoc, two main species co-occurrence groupings (Fig. 3b). Members of the first 
376 group (hereafter referred to as Leptochelia group) included Leptochelia sp. (tanaid), Photis

377 brevipes (gammaridean amphipod), Amphissa columbiana (snail), Spirorbis sp. (calcifying

378 polychaete), Nereis sp. (polychaete worm), Caprella laeviuscula and Caprella natalensis

379 (caprellid amphipods) (Fig. 3b). These taxa positively co-occurred more often than expected by

380 chance or by environmental filtering. They negatively co-occurred with members of the second

381 group, which included a harpacticoid copepod species, Mytilus sp. (mussel), Porcellidium sp.

382 (copepod), Ischyrocerus sp. (gammarid amphipod), and Penidotea resecata (isopod) (hereafter

383 referred to as harpacticoid group). Alvania compacta and Lirularia sp. (snails) positively co-

384 occurred with each other but did not strongly co-occur with members of the two main groups.

385 Remaining taxon pairs did not exhibit strong positive or negative co-occurrence patterns.

386 The two co-occurrence groups were represented at all meadows, suggesting that members

387 from these groups can be simultaneously present (Fig. 3d). However, their abundances tended to

388 negatively co-vary; at meadows JB, HL, CB, RA, EB, DC, and DU, members of the Leptochelia

389 group were most abundant, whereas Harpacticoid group members were most abundant at SS, SA,

390 LH, GB and RB. Meadow HL was an outlier, as it was strongly dominated by Spirorbis sp. The

391 emergent species groupings identified in Fig. 3b do not clearly correspond with geographical

392 structure because the cluster analysis did not group meadows by region membership (black

393 symbols, Fig. 3d). 
a) Quadrat-level co-occurrences

396

397

398

399

400

401

402

403

404

405

406

407

408

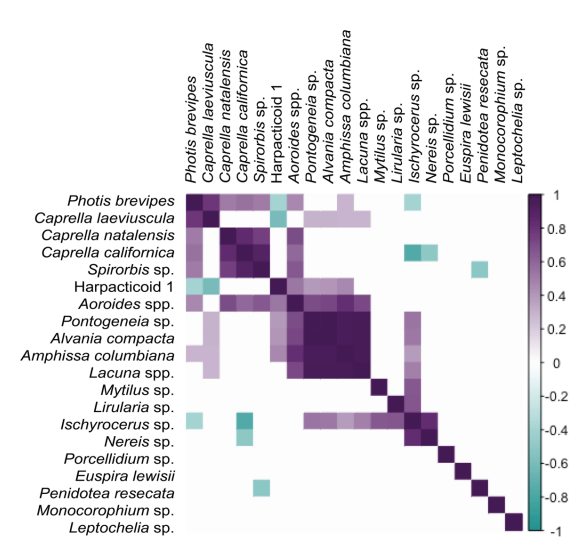

c) Region-level co-occurrences
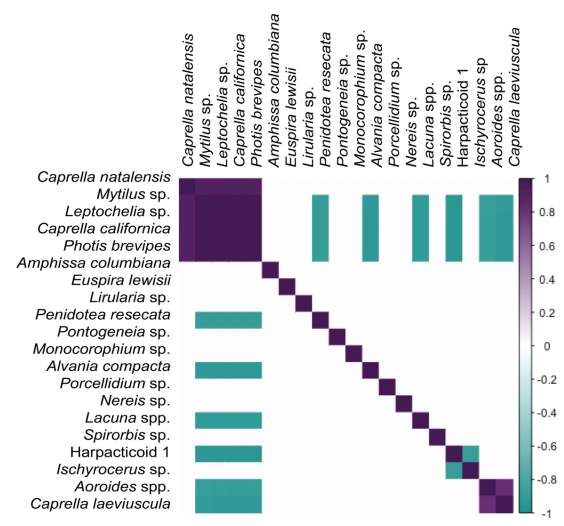

b) Site-level co-occurrences

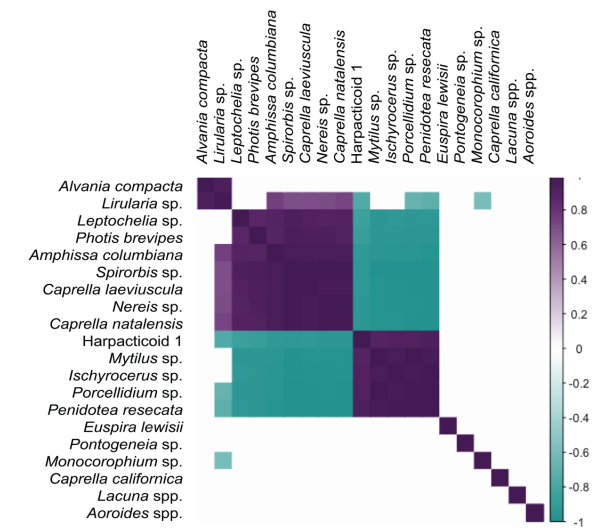

d) Site-level composition (10 most abundant taxa)

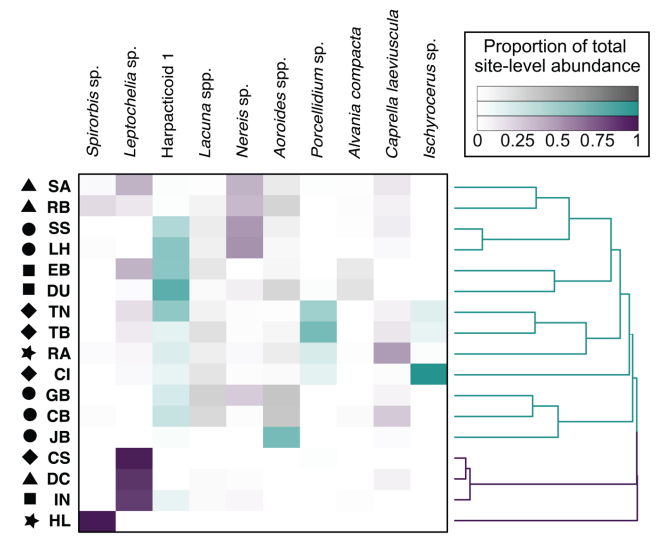

Figure 3. a-c) Correlation plots showing modelled a) quadrat-level, b) site-level, and c) region-level

pairwise co-occurrences after removing the effect of shared responses to the environmental covariates in our model. Only the 20 most abundant species (according to total abundance across the metacommunity or study region) are represented. Purple cells represent positively co-occurring species pairs, and turquoise cells represent negatively co-occurring species pairs. Species names are ordered according to the output of hierarchical clustering with Ward's criterion on pairwise co-occurrence values. d) Heatmap and cluster dendrogram depicting species relative abundance and compositional similarity across sites. Species are ordered by decreasing abundance from left to right. Cell colours correspond to the two main site-level cooccurrence groupings shown in Fig. 3b: purple or "Leptochelia" group, and blue or "Harpacticoid" group. Cell shade strength indicates proportional abundance at a given site (darker means higher relative abundance). The black symbols to the left of the site abbreviations indicate region membership; stars indicate Haida Gwaii sites, diamonds indicate Central Coast sites, triangles indicate Barkley Sound sites, squares indicate Clayoquot Sound sites, and triangles indicate Southern Gulf Islands sites. 


\section{Discussion}

With a biodiversity survey of 17 seagrass meadows across an approximately $1000 \mathrm{~km}$

420 spatial extent, we fitted a joint species distribution model to test hypotheses about the

421 contributions of dispersal, environmental filtering, and species interactions to epifaunal

422 invertebrate abundance and distribution. We also documented at least two previously

423 unrecognized species' associations in the region. This study is the largest (in geographical

424 extent) spatially explicit seagrass-based metacommunity study to our knowledge. We found little

425 support for complete dispersal limitation even across over $1000 \mathrm{~km}$ of coastline, some support

426 for the importance of environmental niche filtering, and co-occurrence patterns that might have

427 arisen from interspecific interactions. Overall, these results suggest that seagrass-associated

428 biodiversity patterns reflect ecological processes spanning local (meadow-scale) to regional

429 scales, and provide additional support for recent findings that eelgrass-associated diversity

430 reflects regional-scale ecological processes in addition to local scale processes (Lefcheck et al.

431 2016, Whippo et al. 2018, Stier et al. 2019, Yeager et al. 2019).

433 Dispersal limitation is unlikely at this regional spatial scale

434 Our findings that: 1) Many species were present in all regions or all meadows; 2) The

435 effect of spatial distance alone did not explain decay in community similarity (Fig. 1b); 3) There

436 was a low contribution of spatial autocorrelation to variance in species abundance (Fig. 2a); and

437 4) Hierarchical cluster analysis did not group meadows according to spatial regions (black

438 symbols in Fig. 3d), all suggest that distance between meadows likely does not confer dispersal

439 limitation preventing populations at distant sites from exchanging individuals. It is however

440 likely that meadows that are physically near each other likely experience higher rates of 
441 exchange in individuals than distant ones, given our observation that dissimilarity increases

442 logarithmically with increased spatial distance. The six taxa that were present at all meadows and

443 an additional ten taxa that were present in all regions had representation from across phyla and

444 life history traits (including rare taxa), suggesting that no single taxonomic group or dispersal

445 strategy had a consistently larger spatial distribution than others. Many meadows were small $(<1$

446 ha), and epifaunal abundances can vary substantially within meadows from year to year (Nelson

447 1997, Douglass et al. 2010) such that local extinctions followed by rescue from nearby

448 populations are likely (Thom et al. 1995). However, the specific pathway that dispersing

449 organisms travelled to arrive at these meadows remains unknown. We used Euclidean distances

450 in our analysis, which preserved the rank order of distances between meadows, but actual

451 distances are likely greater due to oceanographic circulation patterns (Kinlan and Gaines 2003,

452 Mitarai et al. 2008).

453 It is unclear whether distant meadows share taxa because they: 1) Are linked by direct

454 dispersal via oceanographic processes (currents); 2) Are indirectly linked by dispersal via

455 unsampled "stepping-stone" meadows or other habitat types; or 3) Were colonized by

456 populations of the same species in a historical dispersal event, but have not seen the exchange of

457 individuals since (Lefcheck et al. 2016). Seagrass-associated epifauna generally disperse

458 passively between meadows, either through larval transport in currents or by "rafting” on

459 floating pieces of seagrass or macroalgae. Rafts have been observed dispersing benthic and

460 epifaunal invertebrates across phyla (echinoderms, peracarids, molluscs, annelids), and facilitate

461 connectivity between coastal ecosystems across 100s of kilometres (Wichmann et al. 2012).

462 Our inferences are based on an analysis that necessarily emphasized common taxa-

463 either numerically dominant or present in most meadows, or both. We observed seventeen rare 
464 taxa that were found in fewer than $5 \%$ of our samples, often at extremely low abundances (1-2

465 individuals per meadow, or per 6 quadrats) in our survey of over 50000 individuals. The spatial

466 extent of their range could be dispersal-limited for reasons other than prohibitively long travel

467 distances. Populations with low abundances may not disperse in appreciable numbers, and

468 therefore cannot establish populations as readily as abundant species; this is supported by our

469 observation of a positive relationship between range size (the number of meadows a species was

470 observed at) and abundance (Appendix 1: Fig. S4). This highlights a potential bias in studies like

471 ours against detecting dispersal limitation if it is most severe for rare taxa. Increasing the sample

472 size might reduce the risk of this type of bias, but would cost a substantial amount of time

473 associated with invertebrate identification. More efficient biodiversity sampling methods (e.g.

474 eDNA) could overcome this time cost.

476 2017) allows species to colonize sites where local environmental conditions are optimal for

477 growth and reproduction, whereas high dispersal ('dispersal surplus') overrides this, allowing

478 species to persist in habitats that cannot sustain positive population growth without substantial

479 immigration ('mass effects', Mouquet and Loreau 2013). A subset of species was present at all

480 meadows, but their abundances varied (Fig 3d); this pattern may suggest weak mass effects,

481 where dispersal rates are high enough for several taxa to occur at most meadows even if local

482 conditions are suboptimal, but not so high as to completely overwhelm the signature of

483 environmental conditions (Schmida and Wilson 1985, Mouquet and Loreau 2003).

484 Metacommunity theory predicts that such weak mass effects are likely whenever dispersal rates

485 are not limiting (Thompson et al. 2017), particularly in organisms that cannot control their own

486 dispersal (Leibold and Chase 2017) such as seagrass-associated invertebrates. This may explain 
487 the poor model fit in some ubiquitous taxa such as Amphissa columbiana $\left(\mathrm{R}^{2}=0.05\right.$, Appendix

488 1: Table S2). The poor model fit may suggest that these taxa either 1) persist at several meadows

489 even if local environmental conditions are not optimal, due to sufficient immigration, or 2) have

490 broader environmental niches, and thus the environmental gradient observed across the study

491 region is not sufficient to observe clear responses (lower or higher abundances) in these taxa.

492

Regional species abundance patterns suggest weak environmental filtering

We found that differences in local-scale environmental factors account for some

495 differences in invertebrate presence and abundance $(\mathrm{H} 2)$, however there remains a great degree

496 of unexplained variation given the imperfect model fit (mean $\mathrm{R}^{2}=0.27$ ). Abiotic environmental

497 variables had a greater influence on invertebrate distribution and abundance than biotic variables

498 (Fig. 2a). The estimated $\beta$ parameters suggest that, generally speaking, environments that are

499 saltier, have a higher seagrass surface area, smaller annual temperature range, and higher

500 dissolved oxygen levels have higher abundances of many taxa in this study (Fig. 2b). However,

501 there were several exceptions to this trend, highlighting the importance of understanding

502 individual species responses to the environment as opposed to the community as a whole.

503 Overall, we conclude that, at least in taxa for whom the model that had a higher fit (e.g.

504 Leptochelia sp., $\mathrm{R}^{2}=0.52$ ), environmental filtering influences regional patterns in abundance

505 and distribution.

506 Differences in environmental conditions may influence distribution and abundance across

507 the region in one of three ways. First, abiotic variables such as temperature and nutrient

508 availability may influence food availability through primary productivity. This is possible despite

509 the fact that biometric variables (seagrass LAI, biomass) explained a relatively low proportion of 
510 variance in abundance; while these measurements were taken at a single time point during field

511 sampling, the abiotic variables represented annual averages (Assis et al. 2018) and therefore

512 better represent long-term productivity. Second, temperature and salinity may affect species’

513 abundances via environmental tolerance ranges; for example, the isopod Idotea baltica (a

514 relative of $P$. resecata and $P$. montereyensis in our study) experiences significantly lower

515 survival in prolonged exposure to higher temperatures and lower salinities than those in their

516 home habitat (Rugiu et al. 2018). Third, temperature may influence invertebrate metabolic

517 demands, thereby influencing consumption rates and available primary biomass (O'Connor

518 2009). Reduced food availability may increase competition, thus altering the number of

519 individuals a seagrass patch can host.

520 Environmental conditions in temperate seagrass meadows fluctuate seasonally, driving

521 changes in epifaunal community structure throughout the year (Nelson et al. 1979, Wlodarska-

522 Kowalczuk et al. 2014, Whippo et al. 2018). Sampling meadows several times within a year to

523 capture these temporal changes would clarify the importance of the environment if invertebrate

524 assemblages shift with changes in environmental variables following the linear relationships

525 uncovered in our analysis.

527 Species co-occurrence patterns may suggest the importance of interspecific interactions

528 We did not observe strong between-region co-occurrence patterns, suggesting that there

529 do not appear to be clear regional assemblages corresponding with the five subregions in our

530 analysis (Figs. 3c, black symbols in 3d). We also did not observe strong co-occurrence patterns

531 between quadrats, however we did observe strong co-occurrence patterns at the meadow-level

532 spatial scale. The explanation is that meadows had distinct assemblages, and these assemblages 
533 tended to be homogenized within meadows; if two taxa co-occurred in a given meadow (Fig.

$5343 \mathrm{~b}$ ), then they likely co-occurred in all quadrats within the meadow (Fig. 3a). This is consistent

535 with previous findings that epifaunal diversity patterns do not differ from random patterns within

536 meadows (between quadrats) (Whippo et al. 2018).

At the meadow-level, two main co-occurrence groupings showed antagonistic abundance

538 patterns across meadows that could not be explained by spatial structure or shared responses to

539 the environment (Fig. 3d). These co-occurrence groups have not, to our knowledge, been

540 explicitly documented before in this region. While there are multiple possible explanations for

541 non-random species co-occurrence patterns (Connor and Simberloff 1979), we ruled out distance

542 between meadows and measured environmental variables, because co-occurrence values were

543 extracted from residual variation unexplained by the environment or space (Ovaskainen et al.

544 2017). This residual variation therefore likely can be explained by a combination of unmeasured

545 environmental variables, stochasticity, and biotic interactions.

547 in our survey are herbivores or detritivores, and thus may compete for primary (mostly epiphytic

548 algae) biomass. Laboratory experiments have shown that grazing rate and habitat selection in

549 amphipods are altered in the presence of morphologically and functionally similar interspecific

550 competitors (Howard 1985, Brooks and Bell 2001, Beermann et al. 2018). Other species in our

551 study are suspension feeders, and thus may not compete for food but possibly predator-free space

552 or substrate on eelgrass blades.

553 The invertebrate assemblage at a given meadow may have multiple possible

554 compositional states depending on the arrival order of species; this phenomenon is known as

555 priority effects (Fukami et al. 2016, Ke and Letten 2018) and has been documented in marine 
556 fouling communities (Vieira et al. 2018). The antagonistic co-occurrence groupings in our

557 analysis could suggest priority effects at the meadow-level. It is possible that, following seasonal

558 declines in abundance or a disturbance event, the first few populations to increase in abundance

559 or colonize a meadow determine the success of others. Abundance patterns in the sessile

560 calcifying polychaete Spirorbis sp. might demonstrate an example of priority effects. Spirorbis

561 sp. dominated the HL meadow on Haida Gwaii; there were approximately 16300 individuals,

562 several orders of magnitude higher than its abundance elsewhere. Meadow HL also had the

563 lowest taxonomic richness of all 17 meadows (9 species), and we observed fewer micro and

564 macro-epiphytes on eelgrass blades. Instead, the eelgrass was completely covered with Spirorbis

565 sp. This phenomenon has also been observed in Z. marina meadows in Akkeshi-Ko estuary,

566 Japan (Clark et al. 2018) and in Thalassia testudinum meadows in the Northwestern Gulf of

567 Mexico (Dirnberger 1990). Experimental evidence suggests that Spirorbis spp. larvae tend to

568 settle lower in the water column on newer seagrass growth, away from epiphytic algae and

569 previously settled conspecifics (Dirnberger 1990). Settlement rates were determined by

570 planktonic larval density rather than space availability on seagrass blades (Dirnberger 1990).

571 Given this, it is possible that Spirorbis sp. dominates HL from a combination of density-

572 dependent processes (high larval recruitment) and environmental conditions (high salinity, low

573 nitrates). The low epiphyte load on seagrass at HL, whether mostly driven by high Spirorbis sp.

574 densities or by the environment, may explain the low abundance and diversity of all other

575 invertebrate taxa. Overall, the importance of priority effects likely depends on the age,

576 colonization history, and disturbance regime of seagrass meadows.

577 Negative co-occurrence patterns in taxonomic distributions could also reflect the higher

578 abundance of predators at some meadows. Field experiments have shown that changes in 
579 predation pressure by fish, shorebirds, and predatory invertebrates can shift seagrass-associated

580 epifaunal assemblages in a matter of weeks (Amundrud et al. 2015; Huang et al. 2015). Previous

581 studies involving some of the meadows in this analysis (Haida Gwaii, Clayoquot Sound, and

582 Barkley Sound) found high variation (beta diversity) in fish assemblages among meadows

583 (Robinson et al. 2011, Iacarella et al. 2018). A comprehensive survey of fish and bird predators

584 is required to determine the extent to which top-down trophic interactions structure these

585 invertebrate communities. Positive co-occurrence patterns may additionally be a result of

586 positive biotic interactions. An example is Orchomenella recondita - a gammarid amphipod that

587 lives in the gastrovascular cavity of the anemone Anthopleura elegans (Carlton 2007). This

588 species was only recorded at the SS meadow, and specifically only found in quadrats where $A$.

589 elegans was collected with the seagrass shoots.

590 While inferences from data taken at a single time point have limitations, our JDSM

591 approach suggests that there is more to learn about species interactions and the interplay between

592 interactions and dispersal in structuring these communities. The hypothesized processes driving

593 seagrass epifaunal diversity patterns that can be further tested with experiments to test for

594 priority effects or trophic interactions, with population genetics to test for demographic

595 connectivity, or with particle tracking models (e.g. Treml et al. 2008) to estimate dispersal

596 velocity.

598 Conclusion

Seagrasses are important coastal foundation species, valued throughout the world for

600 supporting diverse and productive food webs (Constanza et al. 1997, Williams and Heck 2001).

601 The extent of these ecosystems is declining at rates believed to exceed those of coral reef and 
602 rainforest habitat loss, suggesting major losses of associated biodiversity (Waycott et al. 2009).

603 Our finding that several seagrass-associated faunal taxa across functional groups are consistently

604 present across a fairly large spatial scale is consistent with notion that "the mobile, fast-

605 reproducing, and generally omnipresent animal community is keenly responsive to the presence

606 of habitat" (Lefcheck et al. 2016). Our study suggests that the management of seagrass for its

607 ecosystem services may be more effective if both local and regional processes are considered

608 explicitly in habitat protection plans (Economo 2011).

\section{$610 \quad$ Literature Cited}

611 Aivelo, T. and Norberg, A. 2018. Parasite-microbiota interactions potentially affect intestinal communities in wild mammals. J. Anim. Ecol. 18:438-447.

613 Amundrud, S. L. et al. 2015. Indirect effects of predators control herbivore richness and

614 abundance in a benthic eelgrass (Zostera marina) mesograzer community. - J. Anim. Biol.

$615 \quad 84: 1092-1102$.

616 Assis, J. et al. 2018. Bio-ORACLE v2.0: Extending marine data layers for bioclimatic modelling. - Global Ecol. Biogeogr. 27:277-284.

618 Beermann, J. et al. 2018. Combined effects of predator cues and competition define habitat choice and food consumption of amphipod mesograzers. - Oecologia 186:645-654.

620 Best, R., and Stachowicz, J. J. 2014. Phenotypic and phylogenetic evidence for the role of food and habitat in the assembly of communities of marine amphipods. - Ecolog. 95: 775-786.

622 Berlow, E. L. 1999. Strong effects of weak interactions in ecological communities. - Nature 398: 330-334. 
624 Boström, C. et al. 2006. Seagrass landscapes and their effects on associated fauna: A review. Estuarine, Coastal Shelf Sci. 68: 383-403.

Boström, C. et al. 2010. Invertebrate dispersal and habitat heterogeneity: Expression of biological traits in a seagrass landscape. J. Exp. Mar. Biol. Ecol. 390:106-117.

Bray, J. R. and J. T. Curtis. 1957. An ordination of upland forest communities of southern Wisconsin. - Ecol. Monogr. 27:325-349.

Brooks R.A., and Bell S.S. 2001. Mobile corridors in marine landscapes: Enhancement of faunal exchange at seagrass/sand ecotones. 2001. - J. Exp. Mar. Bio. Ecol. 264: 67-84.

632 Brown, B. L. et al. 2017. Making sense of metacommunities: dispelling the mythology of a metacommunity typology. - Oecologia 183:643-652.

634 Carlton, J. 2007. The Light and Smith Manual: Intertidal invertebrates from Southern California to Oregon. Fourth edition. University of California Press, Oakland, California, USA.

636 Cébrian, J. and Duarte, C. M. 1998. Patterns in leaf herbivory on seagrasses. - Aquat. Bot. 60:67-82.

638 Connor, E. F. and Simberloff, D. 1979. The assembly of species communities: Chance or competition? - Ecology 60:1132-1140.

Costanza, R., et al. 1997. The value of the world's ecosystem services and natural capital. Nature 387:253-260.

642 Cottenie, K. 2005. Integrating environmental and spatial processes in ecological community dynamics. - Ecol. Lett. 8:1175-1182.

644 Duffy J. E. et al. 2015. Biodiversity mediates top-down control in eelgrass ecosystems: A global comparative-experimental approach. - Ecol. Lett. 18:696-705. 
646 Economo, E. P. 2011. Biodiversity conservation in metacommunity networks: Linking pattern and persistence. - Amer. Nat. 177:167-180.

Fukami, T. et al. 2016. A framework for priority effects. - J. Veg. Sci. 27:655-657.

649 Gaines, S. and Roughgarden, J. 1985. Larval settlement rate: A leading determinant of structure in an ecological community of the marine intertidal zone. - Proc. Natl. Acad. Sci.

Gilbert, B. and Bennett, J. R. 2010. Partitioning variation in ecological communities: do the numbers add up? - J. Appl. Ecol. 47:1071-1082.

654 Guillaume Blanchet, F. et al. 2018. HMSC: Hierarchical Modelling of Species Community. R package version. 2.1-2.

656 Gullström, M. et al. 2012. Spatial patterns and environmental correlates in leaf-associated epifaunal assemblages of temperate seagrass (Zostera marina) meadows. - Mar. Biol. $159: 413-425$.

Huang, A. C. et al. 2015. Top-down control by great blue herons Ardea herodias regulates seagrass-associated epifauna. - Oikos 124:1492-1501.

661 Howard, R. K. 1985. Measurements of short-term turnover of epifauna within seagrass beds using an in situ staining method. - Mar. Ecol. Prog. Ser. 22: 163-168.

663 Ke, P. J. and Letten, A. D. 2018. Coexistence theory and the frequency-dependence of priority effects. - Nat. Ecol. Evol. 2:1691-1695.1-7.

665 Kinlan, B. P. and Gaines, S. D. 2003. Propagule dispersal in marine and terrestrial environments: A community perspective. - Ecology 84:2007-2020.

667 Kozloff, E. 1996. Marine Invertebrates of the Pacific Northwest. University of Washington Press, Seattle, Washington, USA. 
669 Leibold, M. A. et al. 2004. The metacommunity concept: A framework for multi-scale community ecology. - Ecol. Lett. 7:601-613.

671 Leibold, M. A. and Chase, J. M. 2017. Metacommunity Ecology, Volume 59. Princeton

672 University Press, Princeton, New Jersey, USA.

673 Lefcheck, J. S. et al. 2016. Faunal Communities Are Invariant to Fragmentation in Experimental 674 Seagrass Landscapes. - PLoS ONE 11(5): e0156550.

675 Levin, S. A. and Paine, R. T. 1974. Disturbance, patch formation, and community structure. Proc. Natl. Acad. Sci. 71:2744-2747.

677 Logue, J. et al. 2011. Empirical approaches to metacommunities: a review and comparison with 678 theory. - Trends Ecol. Evol. 26:482-491.

679 Mitarai, S. D. et al. 2008. A numerical study of stochastic larval settlement in the California Current system. - J. Marine Syst. 69:295-309.

681 Morlon, H. et al. 2008. A general framework for the distance-decay of similarity in ecological communities. - Ecol. Lett. 11:904-917.

683 Mouquet, N. and Loreau, M. 2003. Community patterns in source-sink metacommunities. Amer. Nat. 162:554-557.

685 Nelson, W. G. 1979. An analysis of structural pattern in an eelgrass (Zostera marina L.)

686 amphipod community. - J. exp. Mar. Biol. Ecol. 39:231-264.

687 O’Connor, M. I. 2009. Warming strengthens an herbivore-plant interaction. - Ecology 90:388$688 \quad 398$.

689 Orth, R. J. et al. 1984. Faunal communities in seagrass beds: A review of the influence of plant structure and prey characteristics on predator-prey relationships. - Estuaries 7:339-350. 
691 Ovaskainen, O. et al. 2016a. Uncovering hidden spatial structure in species communities with spatially explicit joint species distribution models. - Methods Ecol. Evol. 7:428-436.

693 Ovaskainen, O. et al. 2016b. Using latent variable models to identify large networks of speciesto-species associations at different spatial scales. - Methods Ecol. Evol. 7:549-555.

695 Ovaskainen, O. et al. 2017. How to make more out of community data? A conceptual framework and its implementation as models and software. - Ecol. Lett. 20:561-576.

R Development Core Team. 2014. R: a language and environment for statistical computing. R Foundation for Statistical Computing. Vienna, Austria. http://www.R-project.org.

Ricklefs, R. E. and Schluter, D. 1993. Species Diversity in Ecological Communities: Historical and Geographical Perspectives. University of Chicago Press, Chicago, Illinois, USA.

Rugiu, L. et al. 2018. Variations in tolerance to climate change in a key littoral herbivore. - Mar. Biol. 165:1-11.

Sala, E. and Graham, M. H. 2002. Community-wide distribution of predator-prey interaction strength in kelp forests. - Proc. Natl. Acad. Sci. 99:3678-3683.

Sand-Jensen. 1977. Effect of epiphytes on eelgrass photosynthesis. - Aquat. Bot. 3:55-63.

Schmida, A. and Wilson, M. V. 1985. Biological determinants of species diversity. - J. Biogeogr. 12:1-20.

Stier, A. C. et al. 2019. Temporal variation in dispersal modifies dispersal-diversity relationships in an experimental seagrass metacommunity. - Mar. Ecol. Prog. Ser. 613:67-76.

710 Thom, R. et al. 1995. Temporal patterns of grazers and vegetation in a temperate seagrass system. - Aquat. Bot. 50:201-205.

712 Thompson, P. L. et al. 2017. Loss of habitat and connectivity erodes species diversity, ecosystem 713 functioning, and stability in metacommunity networks. - Ecography 40:98-108. 
714 Thompson, P. L. et al. 2020. A process-based metacommunity framework linking local and

715 regional scale community ecology. -Ecol. Lett. (In press)

716 Treml, E. A. et al. 2008. Modeling population connectivity by ocean currents: a graph-theoretic 717 approach for marine conservation. - Landsc. Ecol. 23:19-36.

718 Tuomisto, H. et al. 2012. Modelling niche and neutral dynamics: on the ecological interpretation 719 of variation partitioning. - Ecography 35:961-971.

720 Tyberghein, L. et al. 2011. Bio-ORACLE: a global environmental dataset for marine species 721 distribution modelling. - Glob. Ecol. Biogeogr. 21: 272-281.

722 Vieira, E. et al. 2018. Persistence and space preemption explain species-specific founder effects

723 on the organization of marine sessile communities. - Ecology and Evolution 8:3430-3442.

724 Virnstein, R. W., and R. K. Howard. 1987. Motile epifauna of marine macrophytes in the Indian

725 River Lagoon, Florida. II. Comparisons between drift algae and three species of seagrasses.

726 Bulletin of Marine Science 41:13-26.

727 Vellend, M. 2010. Conceptual synthesis in community ecology. - Q. Rev. Biol. 85:183-206.

728 Ward, J. H. 1963. Hierarchical grouping to optimize an objective ounction. - Journal of the

729 American Statistical Association 58:236-244.

730 Waycott, M. et al. 2009. Accelerating loss of seagrasses across the globe threatens coastal

$731 \quad$ ecosystems. - Proc. Natl. Acad. Sci. 106: 12377-12381.

732 Wichmann, C. S. et al. 2012. Floating kelps in Patagonian Fjords: An important vehicle for 733 rafting invertebrates and its relevance for biogeography. - Mar. Biol. 159:2035-2049. 
734 Williams, S. L., and K. L. Heck, Jr. 2001. Seagrass community ecology. Pages 317-337 in M. D.

735 Bertness, M. E. Hay, and S. D. Gaines, editors. Marine community ecology. Sinauer

736 Associates, Sunderland, Massachusetts, USA.

737 Whalen, M. A., et al. 2013. Temporal shifts in top-down vs. bottom-up control of epiphytic algae 738 in a seagrass ecosystem. - Ecology 94:510-520.

739 Whippo, R. et al. 2018. Epifaunal diversity patterns within and among seagrass meadows suggest $740 \quad$ landscape-scale biodiversity processes. - Ecosphere 9:02490.10.1002/ecs2.2490.

741 Wlodarska-Kowalczuk, M. et al. 2014. Evidence of season-dependency in vegetation effects on 742 macrofauna in temperate seagrass meadows (Baltic Sea). - PLoS One 9:e100788.

743 Yamada et al. 2007. Temporal and spatial macrofaunal community changes along a salinity 744 gradient in seagrass meadows of Akkeshi-ko estuary and Akkeshi Bay, northern Japan. $745 \quad$ Hydrobiologia 592: 345-358.

746 Yamada, K. et al. 2014. Environmental and spatial controls of macroinvertebrate functional 747 assemblages in seagrass ecosystems along the Pacific coast of northern Japan. - Glob. Ecol. 748 Cons. 2:47-61.

749 Yeager, L. A. et al. 2019. Trait sensitivities to seagrass fragmentation across spatial scales shape 750 benthic community structure. - J. Anim. Ecol. 88:1743-1754. 